\title{
Genetic Protection Modifications - Moving Beyond the Binary \\ Distinction between Therapy and Enhancement for Human Genome Editing $^{1}$
}

Mikkelsen $\mathrm{R}^{* \S}$, Frederiksen $\mathrm{H}^{* *}$, Gjerris $\mathrm{M}^{*}$, Holst $\mathrm{B}^{* * *}$, Hyttel $\mathrm{P}^{* *}$, Luo $\mathrm{Y}^{* * *}$, Freude $\mathrm{K}^{* *}$, Sand $\varnothing$ e $\mathrm{P}^{* * *}$

* Department of Food and Resource Economics, University of Copenhagen

${ }^{* *}$ Department of Veterinary and Animal Sciences, University of Copenhagen

${ }^{* * *}$ Bioneer $\mathrm{A} / \mathrm{S}$

${ }^{* * * *}$ Lars Bolund Institute of Regenerative Medicine, BGI-Shenzhen \& Department of Biomedicine, Aarhus University

${ }^{\S}$ Corresponding author: Rolighedsvej 25, Building C, $4^{\text {th }}$ Floor, DK-1958 Frederiksberg C, Denmark. E-mail: rbm@ifro.ku.dk. Tel: (+45) 35330735

\begin{abstract}
Current debate and policy surrounding the use of genetic editing in humans often relies on a binary distinction between therapy and human enhancement. In this paper, we argue that this dichotomy fails to take into account perhaps the most significant potential uses of CRISPR-Cas9 gene editing in humans. We argue that genetic treatment of sporadic Alzheimer's disease, breastand ovarian-cancer causing BRCA1/2 mutations and the introduction of HIV resistance in humans should be considered within a new category of genetic protection treatments. We find that if this category is not introduced, life-altering research might be unnecessarily limited by current or future policy. Otherwise ad hoc decisions might be made, which introduce a risk of unforeseen moral costs, and might overlook or fail to address some important opportunities.
\end{abstract}

\footnotetext{
${ }^{1}$ Final publication is available from Mary Ann Liebert, Inc., publishers https://home.liebertpub.com/publications/the-crispr-journal/642
} 
Therapy
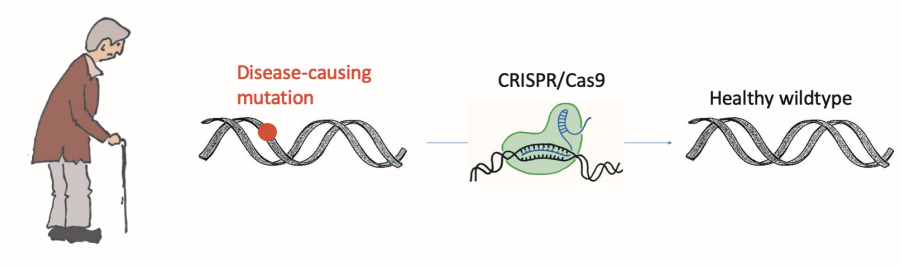
protection modification
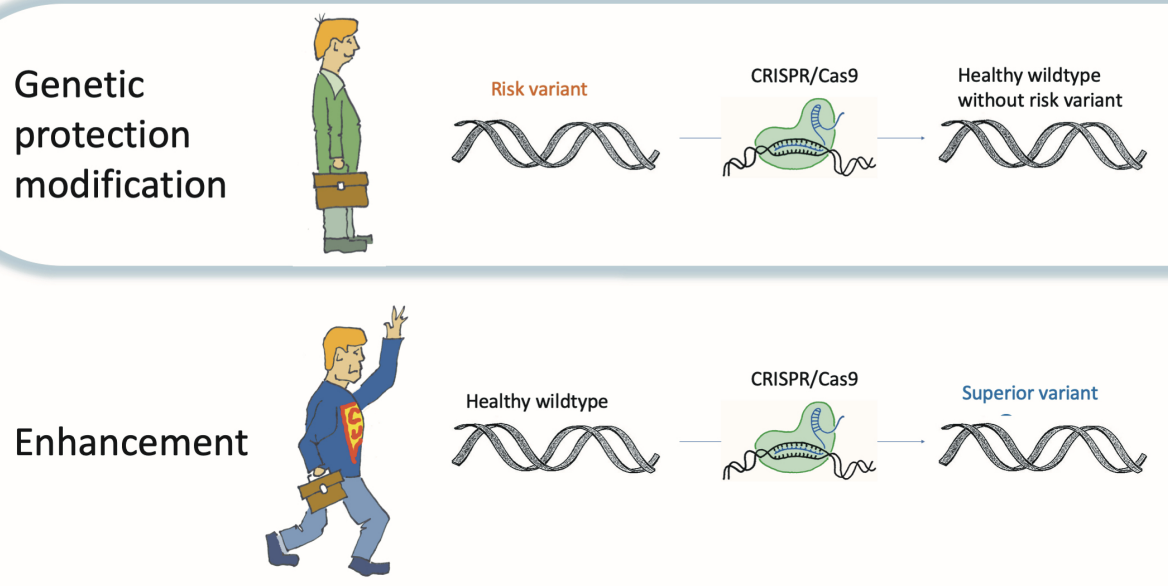

\section{INTRODUCTION}

Gene editing with subsequent transplantation of human cells for treatment purposes has been technically possible for many years, but it was only with the introduction of CRISPR-Cas9 technology in 2012 that it became affordable and precise enough to make widespread use in human medicine a realistic possibility. This led to growing interest in the ethical implications of this and similar technologies.

Traditionally, public debate over gene editing in humans has proceeded on the basis that there is a dichotomy between therapy and enhancement, with the first generally being considered morally legitimate and the second being seen as more problematic. This dichotomy has historical roots in a very broad discussion about human enhancement in general. It can also be found in the academic literature, although here it has been subject to scrutiny and criticism. ${ }^{1-4}$ However, in the following, we will argue that the binary model (as well as some subsequent proposed modifications to this model) ignores a third category, genetic protection modifications, which covers repair of diseasedisposing genetic variants. Recognizing this category, we focus on what may be by far the most significant group of potential applications of gene editing technology. Genetic protection modification has features in common with both therapy and enhancement, and it therefore raises 
important moral dilemmas. Here, we will illustrate this with the case of Alzheimer's Disease, but the same dilemmas arise for many other genetic variants that are associated with disease.

\section{THE BINARY MODEL OF THE ETHICS OF GENETIC MODIFICATION}

Researchers are currently exploring the potential of CRISPR-Cas9 technology in the treatment of human diseases. There is ongoing research into genetic diagnoses, characterizations and potential future somatic or germline treatments of neurodegenerative diseases such as Alzheimer's, Parkinson's Disease, and diabetes, amongst many other diseases. ${ }^{5-7}$ Concurrently (but entirely unrelated to this research), the first in vivo editing and subsequent live birth of human embryos has been accomplished (and widely condemned ${ }^{8-11}$ ) in China.

While policymakers and ethicists have long been aware of the need to set limits on this type of research and treatment, the advent of CRISPR-Cas9 has led to a push to reassess and make explicit previous guidelines on the topic. Both in debate and in policy surrounding the use of genetic modification, the issues have typically been separated into two categories: those raised by genetic therapy and those connected with genetic enhancement. The bifurcation can be observed in many international policy documents and reports, including the Oviedo Convention ${ }^{12}$ and documents issued by the Committee on Human Gene Editing, ${ }^{13}$ the Danish Ethical Council ${ }^{14}$ and the German Ethical Council. ${ }^{15}$

In some respects, this distinction maps onto a much wider and older debate (both in policy and academic circles) over the difference between therapy and enhancement in general. The distinction has been used in reference to genetic enhancement since the late 1980s, when genetic enhancement was criticized by W. F. Anderson as being risky both medically and for society as a whole. ${ }^{16}$ While much of the existing work on the distinction is useful in defining the proper limits of genetic therapy and enhancement, there are limits to the applicability and usefulness of the literature when it comes to the unique ethical challenges presented by genetic treatments.

To expand a little, previous work on the therapy/enhancement distinction was partly motivated by the need to define the proper scope of health care. In this work it was taken for granted that if 
'therapy' could be defined, the scope of health care would thereby be clarified. With this approach, some of the traditional accounts did set effective limits on the obligations of physicians and the health care system in general. ${ }^{1,16}$ But while those limits are far from irrelevant to the case of genetic treatments, the traditional accounts failed to address some of the additional and unique ethical issues associated with genetic enhancement.

Professional domain accounts are an example of this. They draw the line between therapy and enhancement by asking whether a treatment falls within the domain of the health profession in an actual cultural or societal context. ${ }^{1}$ However, while such accounts can be useful in delineating the boundaries between therapy and enhancement that exist in real health care systems, they fail to engage more deeply with what might be considered the ethically problematic questions about the status of genetic enhancements. They explain why genetic enhancements should not be undertaken in a public health care system, but they provide no basis for limiting or calling into question such treatments more generally.

Genetic enhancement attracted ethical condemnation and legal bans long before the types of treatment we now see were even a realistic possibility. Perhaps, as Scully and Rehmann-Sutter have argued, the ethical objections were at least partly due to the longstanding hypothetical nature of the issue (something that is no longer the case, of course) and the wish to justify genetic therapy research and use by clearly separating it from enhancement issues. ${ }^{4}$ Still, an account of the therapy/enhancement distinction that provides a firm foundation for the ethical evaluation of enhancements per se (independently of debates about the scope of health care) is desirable, and perhaps necessary, as we enter a time where genetic enhancement treatments are no longer a science-fiction scenario. Concrete policy-decisions will surely need to be made, after all, not only about the scope of the health care system, but also about the ethical status and legality of genetic enhancements in general.

The following seems to be the best candidate account: Genetic therapy is (best understood as) the treatment of actual diseases that prevent the individual from functioning normally. Enhancement 
creates modifications designed to augment an individual beyond the scope of normal functioning, or to change traits in an individual that are not associated with loss of function.

This distinction is based on the "normal-functioning" definition of disease described by Norman Daniels and James Sabin. ${ }^{17}$ In the wider therapy/enhancement debate it is recognized that the normal functioning account is challenged by preventative treatments. Generally, such treatments involve interventions in normally functioning individuals, so they do not aim at returning an individual to normal functioning. They appear, therefore, to be enhancements. This has led to attempts to modify the account so as to ensure that preventative measures such as vaccines are not considered enhancements.

It is also important to point out that different ways of distinguishing therapy and enhancement exist, but historically the "normal functioning" definition of disease has been highly influential, and thus, a version of this definition is used here to define the two concepts. According to the "normal functioning" definition, a disease is defined in terms of features, or functions, in the individual which depart from "species-typical normal functioning". On this definition, therapy is treatment to restore normal functioning, allowing the patient to re-access a normal range of opportunities, and enhancements are interventions that extend targeted traits or capabilities beyond the normal range. Based on this definition, it can be said that genetic therapies modify gene variants causing phenotypic expressions that are incompatible with species-typical normal functioning (i.e. are diseases). For example, a genetic treatment of sickle cell anaemia might be considered a genetic therapy. Sickle cell anemia is caused by a genetic variation in the beta-globin gene. The mutation causes abnormal shaping of the red blood cells. However, being heterozygotic for this trait is advantageous in regions with a high prevalence of malaria, as individuals without the trait experience increased morbidity and mortality. ${ }^{18}$ Caution is thus essential. Genetic treatments could offer protection for one disease, but increase the risk of other morbidities. Genetic enhancements involve the modification of gene variants associated with traits which, once improved, allow the individual to go beyond normal functioning. Such enhancement may, for example, improve the IQ of a normally functioning individual. 
This binary distinction has gained traction in policy and popular understanding, and it has served as a functional tool to structure ethical decision-making. However, there is a risk that its simplicity fails to capture an important ethical 'middle-ground' containing interventions that cannot be described accurately in terms of therapy or enhancement. Taking this point up, we will argue that a third category, which we have named "genetic protection modifications", is needed in order to understand the full range of future genetic interventions. Furthermore, we will argue that this third category is of special importance because it contains many of the most promising potential genetic interventions, including treatments for at least some forms of Alzheimer's Disease.

\section{NOT ALL TREATMENTS FIT THE BINARY MODEL}

Some genetic variants do not in themselves cause disease, but they make an individual more likely to develop a disease. The treatment of such variants does not in itself constitute therapy, because the genetic variants do not represent a disease or prevent normal functioning. However, modifications to such variants should not be assessed as enhancements either, because they do not enhance the individual beyond normal functioning. The repair or modification of diseasedisposing variants is what we call "genetic protection modification".

An example can be used to illustrate the third category: An intervention to reduce the risk of some forms of Alzheimer's Disease. This disease is a neurodegenerative disorder resulting in gradual decline in memory and cognitive function which ultimately leads to death. It is the most common form of dementia, accounting for more than $60 \%$ of all dementia cases, making it the most common cause of disability in the elderly population. Around 50 million people have Alzheimer's Disease worldwide ${ }^{19}$ (expected to increase to 152 million by $2050^{20}$ ).

Alzheimer's Disease has two forms: familial (fAD) and sporadic (SAD). Besides their hereditary differences, they differ in age of onset, which for $\mathrm{AAD}$ patients is before or around the age of 60, but for SAD patients is above 60 years. Thus, $f A D$ is also known as early-onset Alzheimer's Disease and SAD as late-onset Alzheimer's Disease. fAD is a hereditary form of the disease that is caused by mutations in one of the three genes Presenilin1 (PSEN1), Presenilin2 (PSEN2) and Amyloid Precursor Protein (APP). Around 300 pathogenic mutations causing fAD have been identified in 
one of these three genes. ${ }^{21}$ Individuals with one of these mutations are certain to develop Alzheimer's Disease. Consequently, it can be said that the genetic variants associated with fAD represent a disease: they cause cognitive decline that restricts species-typical functioning.

By contrast, SAD - which accounts for the majority of Alzheimer's cases and has no clear sign of heritability - has been associated with a range of genetic risk factors which, in combination with environmental factors, confer an increased probability of developing Alzheimer's Disease. These factors do not by themselves cause Alzheimer's Disease, but they do increase an individual's risks of developing it, and thus a SAD variant cannot accurately be described as a genetic disease given the definition provided above. Before genome-wide associated studies (GWAS) could be made, the APOE gene was the only known risk factor associated with SAD. However, GWAS studies have uncovered a number of other genes increasing a person's risk of developing SAD, including the TREM2 gene. TREM2 encodes for the TREM2 protein, which plays an anti-inflammatory role in the brain. People with a mutation in this gene have threefold higher risk of developing SAD, ${ }^{22}$ because non-functional TREM2 is believed to lower the ability of microglia to clear amyloid beta from the brain.

Several other genes, such as CLU, SORL1 and CD33, have been identified via GWAS as genetic risk factors (with varying degrees of risk) associated with SAD. ${ }^{23}$ The presence of these genes make a person more susceptible to SAD, especially in combination with environmental factors such as lack of sleep, ${ }^{24}$ alcohol consumption, poor $\operatorname{diet}^{25}$ and pollution. ${ }^{26}$ However, the main risk factors for $S A D$ are age and genetics, so lifestyle and environmental changes will have limited success in preventing SAD. It is also worth noting that although the two types of Alzheimer's Disease vary in age of onset and are associated with different kinds of risk, the resulting disease is the same for both, indicating that similar, if not the same, mechanisms are involved in the development and progression of the disease.

The key point here is that having an fAD variant present in the genome is a disease in progress and this disease will, if it is not prevented from developing, restrict individuals from species-typical normal functioning. SAD, on the other hand, is associated with a set of genetic variants that 
predispose the individual to disease. While these variants are linked to an increased risk of developing Alzheimer's Disease, they are also found in individuals who never develop the disease. In other words, the variants are not completely 'penetrant' - a geneticist's term describing the proportion (or likelihood) of individuals carrying a particular variant whose presence increases the risk that an individual will develop a disease without (alone) actually causing the disease. The modification, or repair, of variants like those involved in SAD fits into the third category of genetic protection treatments proposed in this paper. Under our proposal, genetic therapy treatments restore the normal function of a variant that will cause disease (full penetrance, typically monogenic diseases), whereas genetic protection treatments correct a genetic variant that makes the associated disease more likely to occur (reduced penetrance, typically multifactorial diseases).

\section{A CASE FOR THE POTENTIAL OF PROTECTION TREATMENTS}

CRISPR-Cas9 technology offers a whole new range of possibilities in genetic editing. Currently, the technology is used mainly in research settings, but it is anticipated that genetic modifications will be used in the future either for ex vivo therapy in combination with cell replacement therapies or (even) for in vivo modifications. The in vivo treatment holds great promise if delivery modes can be optimized, as long as the safety issues of gene treatment can be addressed. However, as with many other potential treatments, one of the hardest obstacles to overcome is a precisely targeted and efficient delivery of the gene edited cells to the diseased organs and target cells. In the specific case of treating Alzheimer's Disease with CRISPR-Cas9, any treatment needs to reach affected cells in the brain. This introduces an additional challenge, because the brain is protected by the blood-brain barrier (BBB).

The ex vivo approach, followed by cell replacement with non-diseased cells (gene-modified isogenic cells) would probably involve stem cells or at least neuronal progenitor cells being used for gene editing. Here the most obvious approach would be to perform patient-specific reprogramming of, for example, skin, adipose tissue or blood cells into induced pluripotent stem cells (iPSC). ${ }^{27}$ Subsequently, these could be modified using the CRISPR-Cas9 gene editing method. Using iPSCs would also create an opportunity to first select successfully gene-modified cells and then reproduce those cells, in principle infinitely. Afterwards, extensive ex vivo quality control and 
differentiation into the desired neuronal cell subtype before transplantation into the patient can be performed. While this approach allows unwanted off-target effects to be screened for, the main challenge would be to ensure the quality and efficient delivery of the gene-edited cells.

Another approach would involve direct in vivo gene editing and direct delivery of CRISPR-Cas9 to the brain, targeting the individual cells. As mentioned above, the BBB makes this difficult, but even if the BBB can be passed through, other problems will need to be solved-problems with the distribution of the CRISPR-Cas9 complexes within the brain and the successful targeting of specific cells. One way to overcome these obstacles might be to use virally based delivery of the CRISPRCas9 RNP complex. ${ }^{28}$ The non-pathogenic parvovirus, adeno-associated virus (AAV), seems to be an efficient vector for transgene expression in vivo and has shown promise in the treatment of brain disorders. It should be kept in mind that neurons within the brain are fully differentiated and non-proliferative. This makes it difficult to deliver virus-based CRISPR-Cas9 components in vivo. It also hampers successful transplantation and integration of ex vivo gene-edited cells into the existing brain network. Where in vivo gene editing is concerned, immunogenicity issues associated with the Cas9 protein need to be considered. The most commonly used homologs of Cas 9 are in fact derived from Staphylococcus aureus and Streptococcus pyogenes, two bacteria to which most humans have pre-existing antibodies. ${ }^{29}$ Thus, other homologs of Cas 9 may be needed, or it may be necessary to employ alternative strategies. Thus, both approaches require further study and development, especially where their off-target effects are concerned, although recent technological improvements have significantly lowered the risks here.

\section{WHY A PROTECTION CATEGORY, AND NOT A PREVENTION CATEGORY?}

The incidence of dementia (of which $A D$ is the most common cause) is rising globally, and the number of people suffering from it is expected to continue to grow in the foreseeable future. It can cause serious emotional suffering and put financial pressure on patients, families and caregivers. It also generates significant societal costs: an estimated $\$ 818$ billion worldwide in $2015 .{ }^{20}$ Consequently, genetic protection modifications aimed at diseases such as SAD could assist efforts to relieve human suffering and allow resources currently being swallowed up by $A D$ treatment to be used to support other societal benefits. Such modifications may be ready for use 
in the near future. In itself, this highlights the need to accurately evaluate the ethics of treatments targeting gene variants predisposing to disease. We suggest that recognition of a third category of genetic protection modifications will help to ensure that the ethical evaluation is framed correctly.

The third category should not be confused with existing suggestions in the literature that a "preventative" category should be added to the traditional therapy/enhancement dichotomy. 2,30,31 In the larger therapy/enhancement debate, there has been a recognition that the normal functioning account used here is challenged by preventative treatments. Generally, it can be said that preventative treatments are an intervention in normally functioning individuals, and does not aim at returning an individual to normal functioning. Consequently, such treatments would have to be considered enhancements. This has led to attempts to modify the account in order to ensure that preventative measures such as vaccines are not considered enhancements. Eric Juengst's and similar definitions of preventative treatment separate prevention from enhancement within the normal-functioning definition of disease, but these definitions fail to distinguish disease-disposing variants from genetic disease variants - a distinction that is fundamental to the genetic protection category proposed here. Thus, Juengst would classify treatments of both $F A D$ and SAD as preventative on the basis that the treatment of both variants aims to delay or prevent the development of Alzheimer's Disease. Instead we define genetic treatments of $F A D$ as therapeutic because they address a disease in progress and classify genetic manipulations effected in response to SAD as protection modifications because SAD variants "merely" increase the risk of disease.

The separation of genetic modifications into the three distinct categories of genetic therapy, protection, and enhancement is important if controversial cases are to receive rigorous ethical assessment. The range of potential genetic protection modifications is broad and varied. Of course, the fact that an intervention is a case of genetic protection does not in itself determine its ethical status. Nevertheless, recognition of this category serves to highlight a group of interventions that are different from therapy or enhancement in a morally relevant way. Our view is that genetic protection interventions should be assessed on their individual merits. Our concern is that, if genetic protection is not seen as a distinct category, their moral status may be 
determined inaccurately as a result of their being classified either as enhancements or as therapies. The difficulty of making sound ethical assessments of genetic protection interventions can be illustrated with a recent and highly publicized example. Recently, He Jiankui and his team conducted human experiments that led to the birth of two genetically edited babies. He's intent was to imbue the children with genetic resistance to HIV (leaving aside suggestion that He was aware of the potential cognitive benefits of editing the CCR5 gene). ${ }^{32,33}$ This experiment has been widely condemned. But, in the light of our triadic categorization it can be seen that some criticisms have been more valid than others.

He and his team have been rightly criticised for their lack of understanding of safety and efficacy issues, and for lack of awareness of the wider negative implications of the intervention. ${ }^{34}$ The informed consent process they followed has been called into question, and there is general agreement that their work displayed a worrying disregard for ethical, legal, and policy norms on the clinical use of genetic editing treatments.

However, He and his team has been further criticized for providing a human enhancement treatment to the children in question, ${ }^{8}$ and on our analysis this accusation is unjustified. After all, most people live their entire lives without contracting HIV, or even encountering circumstances where HIV-resistance would benefit them. This means that a genetic modification providing HIVresistance is more correctly categorized as a genetic protection modification: it seeks to remove or mitigate the effects of a genetic variant that predisposes an individual to develop a disease, much like a treatment that delays or reduces the likelihood of developing SAD, or entirely prevents it. This comparison of modifications aimed at SAD and HIV-resistance brings out a difficulty in the moral assessment of genetic protection modifications: it enables us to see that some protection modifications provoke the same moral worries as genetic enhancement (HIV protection), while others are superficially similar to genetic therapies (SAD). It is only with the introduction of the genetic protection category that the corollaries between these two cases become apparent. This underlines the need for a more sophisticated approach than is available in the binary therapy/enhancement account. In their report on human genome editing, the National Academies of Science suggest that the commonness, or frequency, of a converted sequence should play a role 
in determinations of the permissibility of converting that sequence through genetic editing. ${ }^{13}$ However, following our analysis, the commonness of a sequence should not be treated as an index of the justifiability of the intervention. Instead, the focus should be on the roles the pre- and postconverted sequence play in causing or contributing to loss of normal functioning.

Without the third category, genetic protection modifications are likely to be categorized as enhancements. This could hold back research into genetic protection and potentially have a significant negative impact on human lives. The genetic treatments affected might include those for mutations such as $B R C A 1 / 2$, which also fall into the genetic protection category. While mutations in these genes increase the risk of an individual developing cancer by a significant amount (e.g. by the age of 80 there is a $69-72 \%$ risk of a woman having developed breast cancer and $17-44 \%$ risk of having developed ovarian cancer ${ }^{35}$ ), they do not count as a genetic disease in themselves. Rather they increase the risk of disease. In essence, because the variant is not completely penetrant, it cannot be said that a carrier of it is suffering from a disease until the individual is actually diagnosed with cancer. In practice, it is perhaps likely that the treatment of the mutation in the specific case of the $B R C A 1 / 2$ would be considered a form of genetic therapy within the current therapy/enhancement model - essentially because of the very high risk of an affected individual developing the disease. However, on our analysis this would be an ad hoc solution, and one that would open the possibility of including any number of disease-disposing variants. Moreover, those whose thinking is guided by the current two-category model might well argue that in principle genetic modification of individuals with $B R C A 1 / 2$ mutations ought to be classified as enhancements and therefore restricted. The potential for such unintuitive outcomes, and thus the need for ad hoc solutions, is in our view significant. The genetic protection model does not suffer from this issue.

In short, then, if policy and regulation are based on the binary distinction between therapy and enhancement there is a risk either that research into areas such as SAD and BRCA1/2 mutation will be limited because they are classified as enhancement, or that ad hoc exceptions will have to be made to policies without a systematic basis. This could mean that research is limited, and treatments of widespread and life-altering or life-threatening diseases will not be developed. On 
the other hand, if exemptions are made too freely there is a risk that genetic modifications could carry unforeseen moral costs including, but not limited to: unintended impacts on public health system costs or prioritization; social inequality due to lack of access to treatments; or increased valuation of humans based on individual traits rather than common origin. In extreme cases, such exemptions might even confer comparative advantage on recipients which directly disadvantage individuals without the modification. The genetic protection category makes it possible to maintain strict limits on enhancement proper, because modifications of disease-disposing variants are removed from this category and considered separately. Limitations on research and treatments for, for example, $B R C A 1 / 2$ mutations can then be considered on their individual merits rather than as exceptions to general rules limiting enhancement. Ultimately, this should lead to better decision-making.

It is also worth noting that genetic protection modifications present some unique ethical issues that are not found in traditional genetic enhancement or therapy. Since a therapy aims at disease variants, treatment of the variant itself is the only option for a cure. However, many environmental factors are often involved when a variant associated with disease develops into actual disease, and a significant number of these factors are associated with societal, economic and cultural disparities. In these cases, proponents of genetic modification should be aware that a "treatment solution" might serve only to mask what is really a consequence of social injustice or human suffering in general, especially because genetic protection modifications will in some cases be costly, dangerous, or invasive. Genetic protection modifications are intended to improve the lives of individuals, and in view of this a comprehensive assessment of the risks associated with genetic modification versus other treatment options should be carried out. As Erik Parens has pointed out, our possession of new medical means to reach our intended ends "may incline us to ignore the complex social roots of the suffering of individuals". ${ }^{2}$ Seen through this lens, genetic protection modifications might have more in common with the types of preventative measures enacted through public health policy than they do with genetic therapy. While a genetic therapy aims to cure an existing disease, genetic protection modifications aim to prevent diseases from developing in the first place. Consequently, these modifications should be weighed against other preventative measures that might be taken with the same goal in mind. As pointed out above, in 
the case of SAD it has been shown that diet, alcohol consumption and other factors all play a role in the risk of developing the disease, along with genetic factors.

A successful response to these issues will provide a range of individual and societal benefits above and beyond mitigation of the risk of developing AD. Prima facie, this suggests that public health interventions should be prioritized over genetic protection modifications. However, this prioritization should be balanced against other concerns. Public health interventions are often criticized for being paternalistic in nature. ${ }^{36}$ They can require significant intervention in the lives of individuals and run counter to societal or cultural norms - an issue that is likely to be less serious when genetic protection treatments are used. Consequently, genetic protection modification should be seen as just one of a range of tools that can be employed to address the health and well-being of individuals, and a tool, moreover, that will need to be selectively employed based on the context and intended results.

Current policy and public debate on genetic editing treatments often revolves around a binary distinction between therapy and enhancement, where the former is considered morally legitimate and the latter unethical. This distinction is also present in parts of the academic literature on the subject, although it has been subject to significantly more scrutiny here. While attempts have been made to move discussion away from this polarization, we suggest that a new three-category approach based on the notion of normal functioning would be fruitful. Such an approach would recognize the unique function of genetic variations that are not completely penetrant, and thus disease-disposing rather than disease-causing. These variants might very well offer the greatest potential for genetic treatment, yet the treatments in question cannot be properly categorized as therapy or enhancement (as they would need to be in the current dichotomy), nor are they readily accommodated in previous attempts to address prevention with the normal functioning account. On our account, the category of genetic therapy should cover treatments for disease-causing variants, while the category of enhancement should contain treatments that edit a healthy wildtype in order to engineer a superior variant. This leaves room for the novel genetic protection category we are proposing: A category covering treatments that seek to edit disease-disposing variants in order to give individuals healthy wildtypes. This three-category approach would allow 
society to regulate enhancements with a significant potential to disrupt or damage existing societal, economic or cultural structures rigorously, and to deal with the unique ethical challenges presented by genetic protection modifications separately. 


\section{ACKNOWLEDGMENTS}

We would like to thank Paul Robinson for thorough editing of the language in this manuscript. We would also like to thank the anonymous referees for their thorough comments which we believe have helped to greatly improve the final paper. The work was funded by the Innovation Foundation Demark (BrainStem \& NeuroStem). 


\section{REFERENCES}

1. Juengst E, Moseley D. Human Enhancement. The Stanford Encyclopedia of Philosophy. Summer 2019 Edition. 2019. https://plato.stanford.edu/archives/sum2019/entries/enhancement/

2. Parens E. Is Better Always Good?: The Enhancement Project. Hastings Cent Rep. 1998;28(1):s1-

17. DOI: $10.2307 / 3527981$

3. Miller FG, Brody H, Chung KC. Cosmetic Surgery and the Internal Morality of Medicine. Camb Q Healthc Ethic. 2000;9(3):353-64. DOI: 10.1017/s0963180100903074

4. Scully J, Rehmann-Sutter C. When Norms Normalize: The Case of Genetic "Enhancement." Hum Gene Ther. 2001;12(1):87-95. DOI: 10.1089/104303401451004

5. Pires C, Hall V, Freude K. Human Induced Pluripotent Stem Cells and their Derivatives for Disease Modeling and Therapeutic Applications in Alzheimer's Disease. In: Alzheimer's Disease. SM Group, Dover, DE. 2016.

6. Ochalek A, Mihalik B, Avci HX, Chandrasekaran A, Téglási A, Bock I, et al. Neurons derived from sporadic Alzheimer's disease iPSCs reveal elevated TAU hyperphosphorylation, increased amyloid levels, and GSK3B activation. Alzheimer's Res Ther. 2017;9(1):90. DOI: 10.1186/s13195-017-0317-z

7. Poon A, Zhang Y, Chandrasekaran A, Phanthong P, Schmid B, Nielsen TT, et al. Modeling neurodegenerative diseases with patient-derived induced pluripotent cells: Possibilities and challenges. New Biotechnol. 2017;39(Pt B):190-8. DOI: 10.1016/j.nbt.2017.05.009

8. Kolata G, Belluck P. New York Times. Why Are Scientists So Upset About the First Crispr Babies? https://nyti.ms/2E41TYg. Last accessed 11/06/2019 
9. Collins FS, National Institutes of Health. Statement on Claim of First Gene-Edited Babies by Chinese Researcher. https://www.nih.gov/about-nih/who-we-are/nihdirector/statements/statement-claim-first-gene-edited-babies-chinese-researcher. Last accessed $11 / 06 / 2019$

10. Ramzy A, Wee S. Scientist Who Edited Babies' Genes Is Likely to Face Charges in China. https://nyti.ms/2S0419x. Last accessed 11/06/2019.

11. Cyranoski D. CRISPR-baby scientist fails to satisfy critics. Nature. 2018;564:13-14. DOI: 10.1038/d41586-018-07573-w

12. Hendriks A. Convention for the Protection of Human Rights and Dignity of the Human Being with Regard to the Application of Biology and Medicine: Convention on Human Rights and Biomedicine. Eur J Health Law. 1997;4(1):89-100. DOI: 10.1163/15718099720521896

13. National Academies of Sciences, Engineering, and Medicine. Human Genome Editing: Science, Ethics, and Governance: The National Academies Press. Washington, DC. 2017 DOI:

$10.17226 / 24623$

14. Danish Ethical Council. Udtalelse fra Det Etiske Råd om genetisk modifikation af kommende mennesker. Copenhagen, DK: Det Etiske Råd. 2016

15. German Ethics Council. Germline intervention in the human embryo. Berlin, DE: Deutscher Ethikrat. 2017. DOI: 10.1101/181255

16. Anderson WF. Human Gene Therapy: Why Draw a Line? J Med Philos. 1989;14(6):681-93. DOI: 10.1093/jmp/14.6.681

17. Sabin JE, Daniels N. Determining "Medical Necessity" in Mental Health Practice. Hastings Cent 
Rep. 1994;24(6):5-13. DOI: 10.2307/3563458

18. Ashley-Koch A, Yang Q, Olney R. Sickle Hemoglobin ( $\mathrm{Hb} \mathrm{S}$ ) Allele and Sickle Cell Disease: A HuGE Review. Am J Epidemiol. 2000;151(9):839-45. DOI: 10.1093/oxfordjournals.aje.a010288

19. Alzheimer's Association. 2018 Alzheimer's disease facts and figures. Alzheimers Dement (N Y). 2018;14(3):367-429. DOI: 10.1016/j.jalz.2018.02.001

20. World Health Organization. Dementia. https://www.who.int/news-room/factsheets/detail/dementia. Last accessed 11/06/2019

21. Alzforum. Alzheimer's Mutations. https://www.alzforum.org/mutations. Last accessed $11 / 06 / 2019$

22. Jonsson T, Stefansson H, Steinberg S, et al. Variant of TREM2 Associated with the Risk of Alzheimer's Disease. N Engl J Med. 2013;368(2):107-16. DOI: 10.1056/nejmoa1211103

23. Giri M, Lü Y, Zhang M. Genes associated with Alzheimer's disease: an overview and current status. Clin Interv Aging. 2016;11:665-81. DOI: 10.2147/cia.s105769

24. Hurtley SM. Sleep may protect the brain from AD. Science. 2019;363(6429):831-2. DOI: 10.1126/science.363.6429.831-h

25. Grant WB, Campbell A, Itzhaki RF, Savory J. The significance of environmental factors in the etiology of Alzheimer's disease. J Alzheimer's Dis. 2002;4(3):179-89. DOI: 10.3233/jad-2002-4308

26. Chin-Chan M, Navarro-Yepes J, Quintanilla-Vega B. Environmental pollutants as risk factors for neurodegenerative disorders: Alzheimer and Parkinson diseases. Front Cell Neurosci. 2015;9:124. DOI: $10.3389 /$ fncel.2015.00124 
27. Rasmussen MA, Holst B, Tümer Z, Johnsen MG, Zhou S, Stummann TC, et al. Transient p53 Suppression Increases Reprogramming of Human Fibroblasts without Affecting Apoptosis and DNA Damage. Stem Cell Rep. 2014;3(3):404-13. DOI: 10.1016/j.stemcr.2014.07.006

28. Dong X. Current Strategies for Brain Drug Delivery. Theranostics. 2018;8(6):1481-93. DOI: $10.7150 /$ thno. 21254

29. Crudele JM, Chamberlain JS. Cas9 immunity creates challenges for CRISPR gene editing therapies. Nat Commun. 2018;9(1):3497. DOI: 10.1038/s41467-018-05843-9

30. So D, Kleiderman E, Touré SB, Joly Y. Disease Resistance and the Definition of Genetic Enhancement. Frontiers Genetics. 2017;8:40. DOI: 10.3389/fgene.2017.00040

31. Juengst E. Can Enhancement Be Distinguished from Prevention in Genetic Medicine? J Med Philos. 1997;22(2):125-42. DOI: 10.1093/jmp/22.2.125

32. Regalado A. MIT Technology Review. China's CRISPR twins might have had their brains inadvertently enhanced. https://www.technologyreview.com/s/612997/the-crispr-twins-hadtheir-brains-altered/. Last accessed 11/06/2019

33. Zhou M, Greenhill S, Huang S, et al. CCR5 is a suppressor for cortical plasticity and hippocampal learning and memory. Elife. 2016;5:e20985. DOI: 10.7554/elife.20985

34. Wei X, Nielsen R. CCR5- $\Delta 32$ is deleterious in the homozygous state in humans. Nat Med. 2019;25(6):909-910. DOI: 10.1038/s41591-019-0459-6

35. Kuchenbaecker KB, Hopper JL, Barnes DR, et al. Risks of Breast, Ovarian, and Contralateral Breast Cancer for BRCA1 and BRCA2 Mutation Carriers. Jama. 2017;317(23):2402-16. DOI: 10.1001/jama.2017.7112 
36. Faden R, Shebaya S. Public Health Ethics. The Stanford Encyclopedia of Philosophy. Winter 2016 Edition. 2016. https://plato.stanford.edu/archives/win2016/entries/publichealth-ethics/ 